\title{
The softer, the faster
}

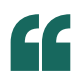

Our findings
open new

ways to target

biological

tissues using

soft particles
Nanoparticles keep surprising us with behaviours that contradict our intuition based on our understanding of bulk materials. Now, Xavier Banquy, Vincent Martinez and colleagues report in Nature Communications that soft nanoparticles diffuse through a polymeric medium much faster than solid nanoparticles of the same size.

Because of their very small size, nanoparticles have been identified as ideal carriers of small molecules in many technological applications from water desalination to medicine - as they can penetrate through intricate, porous membranes. However, if the pores of the membrane are smaller than the nanoparticles themselves, then their diffusion becomes very hard to predict. As drug delivery systems, large nanoparticles have been found to be better and more stable carriers than small nanoparticles, but these qualities are counterbalanced by the fact that penetration into the deep tissues is inhibited by size. "Several design rules have been established to improve circulation time or targeting efficiency of the nanocarriers," explains Banquy. "For example, polymer coatings are often used to avoid rapid elimination of nanocarriers from blood circulation. In the race to develop nanocarriers with longer circulation time and deeper penetration into the tissues, scientists have found that soft nanoparticles perform much better than the hard ones."

Banquy and colleagues wanted to provide fundamental insights into the diffusion of soft versus hard nanoparticles and used a biomimetic system to analyse the forces at play in this phenomenon. The group compared the diffusion behaviour in agarose of soft, highly deformable hydrogel nanoparticles made of poly( $N$-iso-propylacrylamide) to that of hard gold or polystyrene nanoparticles. By changing the concentration of the agarose in water, it is possible to change the viscosity of the solution from liquid to gel, effectively mimicking the different viscosity of biological media. The diffusion of soft and hard nanoparticles of different sizes in agarose solutions and gels was analysed using differential dynamic microscopy (DDM). This technique is normally used to study soft matter and uses video microscopy to generate a differential image correlation function from which information about the dynamics and

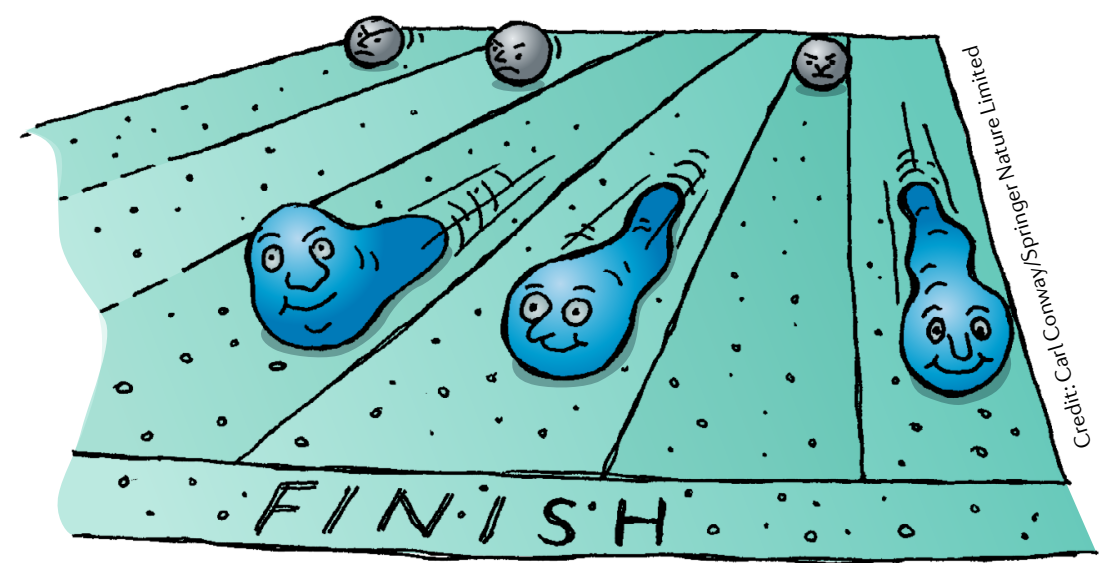

structure of the nanoparticles can be extracted.

The experimental observations confirmed that the diffusion of both soft and hard nanoparticles of similar size was impeded by increasing the viscosity of the agarose solution. The surprising finding, however, was that soft nanoparticles diffuse up to two orders of magnitude faster through the agarose gel than the hard nanoparticles. Comparisons of the experimental observations to trends arising from theoretical models revealed that the faster dynamics of the soft nanoparticles are possible because of their ability to shrink when confined in a dense medium. However, the size reduction of the soft nanoparticles is not due to the osmotic pressure exerted by the medium but is the result of long-range electrostatic interactions between the soft nanoparticle and the agarose gel. Both the agarose fibres and the soft nanoparticles are negatively charged, and it is the consequent electrostatic repulsion that induces a change in the particle size.

"Our findings open new ways to target biological tissues using soft particles, because faster diffusion in a dense porous medium implies deeper penetration," remarks Banquy. "By tuning the mechanical properties of nanoparticles, it is possible to enhance their penetration into softer or denser tissues. This concept can help improve the design of drug delivery systems to target, for example, dense tumours, which are known to resist standard chemotherapeutic treatments," he concludes.

Gabriella Graziano

ORIGINAL ARTICLE Latreille, P.-L. et al. Spontaneous shrinking of soft nanoparticles boosts their diffusion in confined media. Nat. Commun. 10, 4294 (2019) 